\title{
EFEITO DO NÚMERO E DA IDADE DE OVOS DE Stenoma catenifer NO PARASITISMO POR Trichogramma pretiosum
}

\author{
Dori Edson Nava ${ }^{1^{*}}$, José Roberto Postali Parra ${ }^{1}$
}

\section{RESUMO}

A utilização de parasitóides de ovos pode ser uma alternativa viável no controle de Stenoma catenifer Walsingham, 1912, considerada a principal praga do abacateiro (Persea americana Mill.). O objetivo deste trabalho foi determinar o número e a idade de ovos de $S$. catenifer no parasitismo por Trichogramma pretiosum. Para a determinar a capacidade de parasitismo de $T$. pretiosum foram oferecidos $10,20,30,40$ e 50 ovos da praga para cada parasitóide. Para determinar a preferência pela idade hospedeira foram utilizados ovos de 12, 36, 60 e 84 horas de desenvolvimento. A relação ideal para se obter o maior parasitismo, em laboratório foi de 1 fêmea de $T$. pretiosum para 20-30 ovos de $S$. catenifer, por um período de exposição de 24 horas. Os ovos de $S$. catenifer com até 60 horas de idade são os mais indicados para parasitismo por T. pretiosum.

Palavras-chave: Parasitóide de ovos, Persea americana, controle biológico.

${ }^{1}$ Depto. de Entomologia, Fitopatologia e Zoologia Agrícola - USP/ESALQ, C.P. 9 CEP 13418-310 - Piracicaba, SP. *nava@esalqu.usp.br 


\title{
EFFECT OF NUMBER AND AGE OF Stenoma catenifer EGGS ON THE PARASITISM OF Trichogramma pretiosum
}

\begin{abstract}
Egg parasitoids can be a feasible alternative in the control of Stenoma catenifer, considered a key pest of avocado (Persea americana Mill.). This study aimed at determining the number and age of $S$. catenifer eggs parasitized by Trichogramma pretiosum. In order to determine parasitism capacity, 10,20,30, 40 and 50 eggs were offered to each parasitoid. In order to determine host age preferences, 12, 36, 60 and 84hour old eggs were utilized. The ideal proportion for best parasitism under laboratory condition was $1 \mathrm{~T}$. pretiosum female to 20-30 S. catenifer eggs, through a 24-hour exposition period. $S$. catenifer eggs up to 60 -hour old are more suitable to parasitism by $T$. pretiosum.
\end{abstract}

Key words: Egg parasitoid, Persea americana, biological control

\section{INTRODUÇÃO}

Nos últimos anos, a produção de frutas destinadas à exportação (melão, uva de mesa, maçã, citros etc.), principalmente para o mercado europeu aumentou consideravelmente, diferentemente da exportação de abacates que vem diminuindo ano a ano (FNP, 2004). Dentre os vários fatores que dificultam as exportações, pode ser destacada a broca-doabacate, Stenoma catenifer Walsingham 1912 (Lepidoptera: Elachistidae) considerada praga quarentenária em vários países, entre eles, os EUA (Wolfenbarger \& Colburn, 1979). Esta praga tornou-se, nos últimos anos, uma seria ameaça à produção de abacates no Brasil, podendo acarretar perdas totais (Hohmann \& Meneguim, 1993). A falta de métodos eficientes de controle tem levado muitos produtores a abandonarem os pomares ou 
substituí-los por outras culturas, já que as características biológicas e a falta de métodos de monitoramento do inseto são fatores que dificultam a aplicação de inseticidas, principal medida de controle utilizada. Neste contexto, a utilização do controle biológico poderá ser mais efetivo, levando em considerações as características da frutífera e da praga.

O controle biológico de insetos pragas em frutíferas tem sido uma alternativa viável em várias regiões do mundo. Dentre os insetos entomófagos da família Trichogrammatidae, os gêneros Trichogramma e Trichogrammatoidea, de características biológicas semelhantes, destacamse como os mais utilizados para o controle de pragas agrícolas da ordem Lepidoptera.

Estudos de levantamento de espécies de Trichogramma parasitando ovos de lepidópteros pragas, em abacateiro, têm demonstrado grande potencial para o controle biológico (Honda \& Luck, 2001; Oatman et al., 1983). Em levantamentos realizados no município de Arapongas, estado do Paraná, foi registrada uma alta ocorrência natural de parasitismo por T. pretiosum e Trichogrammatoidea annulata De Santis, sobre ovos de S. catenifer, chegando a $40 \%$ de ovos parasitados (Hohmann \& Meneguim, 1993). Desta forma, o objetivo deste trabalho foi determinar o número e a idade dos ovos de $S$. catenifer para o parasitismo de Trichogramma pretiosum visando fornecer subsídios para a criação massal do inseto em laboratório.

\section{MATERIAL E MÉTODOS}

Criação de $S$. catenifer. A broca-do-abacate foi criada em sementes de abacate, segundo técnica descrita por Nava \& Parra (2005). Para a obtenção dos ovos, 10 casais foram colocados em gaiolas, constituídas de tubos de ,PVC $(23,0 \times 15,0 \mathrm{~cm})$, revestidas internamente com papel toalha com depressões, que serviu como substrato para a oviposição (Nava et al., 2005). 
Criação de T. pretiosum. O parasitóide foi criado sobre ovos do hospedeiro alternativo Anagasta kuehniella (Zeller), mantido em dieta à base de farinha de trigo integral (97\%) e levedura de cerveja (3\%), de acordo com metodologia proposta por Parra (1997). Os ovos de A. kuehniella foram colados em cartolina azul $(8,0 \times 2,0 \mathrm{~cm})$, por meio de goma arábica diluída em água a $50 \%$ e esterilizados sob lâmpada germicida durante 45 minutos. Os ovos fixados nas cartelas foram submetidos ao parasitismo, em tubos de vidro $(8,5 \times 2,5 \mathrm{~cm})$, por, no máximo, 12 horas e os parasitóides foram alimentados com uma gotícula de mel depositada na parede do tubo. Para o desenvolvimento, tanto das fases imaturas como dos adultos, utilizaram-se câmaras climatizadas com temperatura de $25 \pm 1^{\circ} \mathrm{C}$, umidade relativa de 70 $\pm 10 \%$ e fotofase de 14 horas.

Definição do número de ovos de $S$. catenifer a ser oferecido por parasitóide. Este experimento foi realizado com o objetivo de se evitar o superparasitismo ou para que não seja subestimado o potencial dos parasitóides em experimentos de laboratório. Desta forma, em tubos de vidro $(7,3 \times 1,0 \mathrm{~cm})$, foram oferecidos $10,20,30,40$ e 50 ovos da broca-doabacate para cada fêmea de $T$. pretiosum. As fềmeas foram alimentadas com uma gotícula de mel puro e os tubos vedados com filme plástico de PVC. Após 24 horas, as fêmeas foram retiradas e os ovos foram mantidos em câmaras climatizadas com temperatura de $25 \pm 1^{\circ} \mathrm{C}$, umidade relativa de 70 $\pm 10 \%$ e fotofase de 14 horas, até o escurecimento dos mesmos, indicativo de parasitismo. Os resultados obtidos foram transformados em porcentagem de parasitismo. Foram utilizados cinco tratamentos (número de ovos) e 15 repetições (fềmeas). 
Efeito da idade do ovo no parasitismo por $S$. catenifer. Fêmeas de $T$. pretiosum foram individualizadas em tubos de vidro $(7,3 \times 1,0 \mathrm{~cm})$ contendo na lateral interna uma gotícula de mel puro, sendo o número de ovos da broca-do-abacate definido no experimento anterior. Os tubos foram vedados com filme plástico de PVC e colocados em uma grade de metal. Após 24 horas de parasitismo, as fềmeas foram retiradas dos tubos e os ovos foram colocados em câmaras climatizadas com temperatura de $25 \pm 1^{\circ} \mathrm{C}$, umidade relativa de $70 \pm 10 \%$ e fotofase de 14 horas. Por ocasião do escurecimento dos ovos, portanto, próximo da emergência dos parasitóides, foi avaliada a porcentagem de parasitismo.

Foram testados ovos de 12, 36, 60 e 84 horas de idade para avaliar o parasitismo de T. pretiosum, totalizando quatro tratamentos (idade dos ovos) e 15 repetições (fêmeas).

Análise dos dados. $O$ delineamento experimental foi inteiramente casualizado. Os dados de porcentagem de parasitismo foram transformados em $\sqrt{\mathrm{x}}$ e submetidos à análise de variância e as médias comparadas pelo teste de Tukey $(\mathrm{P} \leq 0,05)$.

\section{RESULTADOS E DISCUSSÃO}

Definição do número de ovos de $S$. catenifer a ser oferecido para cada parasitóide. Fêmeas de Trichogramma pretiosum foram capazes de parasitar de 9,33 a 23,50 ovos de $S$. catenifer, durante 24 horas (Tabela 1). Observou-se que, quando foram oferecidos 10 ovos de $S$. catenifer, o parasitismo foi de 9,33 ovos, diferindo significativamente de quando foram oferecidos 20, 30, 40 e 50 ovos, que corresponderam a 18,0;23,5;21,8 e 22,5 ovos parasitados, respectivamente (Tabela 1). A determinação da necessidade de aproximadamente 20 a 30 ovos de $S$. catenifer para cada 
fêmea de $T$. pretiosum durante 24 horas, está próxima do número utilizado por Pratissoli \& Parra (2001), para a seleção de linhagens de Trichogramma, visando ao controle de Phthorimaea operculella (Zeller) e de Tuta absoluta (Meyrick), pois os referidos autores ofereceram 25 ovos do hospedeiro para as duas espécies de traças. Para Ecdytolopha aurantiana (Lima), Garcia (1998) utilizou 30 ovos para cada parasitóide.

Esta diferença no número de ovos, encontrada no presente trabalho, com os da literatura, pode estar relacionada ao tamanho dos hospedeiros, já que os mesmos, fornecem ao parasitóide uma medida aproximada da quantidade de nutrientes contida no seu interior. Normalmente, hospedeiros maiores permitem o desenvolvimento de mais de um parasitóide por ovo, levando a uma menor quantidade de ovos parasitados (Gomes, 1997). Desta forma, o oferecimento de 20 a 30 ovos de $S$. catenifer durante 24 horas é uma quantidade aparentemente adequada para que não seja subestimado o parasitismo e nem ocorra superparasitismo.

Efeito da idade do ovo no parasitismo por $T$. pretiosum. Observou-se que ovos de $S$. catenifer com 12, 36 e 60 horas foram igualmente parasitados por T. pretiosum, correspondendo a 74,6; 63,7 e 68,4\%, respectivamente, que por sua vez, diferiram significativamente daqueles com 84 horas, que apresentaram um parasitismo de 46,8\% (Figura 1). Esta queda na porcentagem de parasitismo de ovos em estágios embrionários mais desenvolvidos, também foi demonstrado por Lopes \& Parra (1991) e Reznik et al. (1997). Segundo Vinson (1997), este comportamento pode estar relacionado a possiveis alterações na composição dos ovos, pois, no desenvolvimento embrionário, os de nutrientes de reserva passam a tecidos mais complexos quimicamente. No entanto, há divergências a respeito da qualidade do desenvolvimento dos parasitóides em ovos de diferentes idades, pois o recurso nutricional e a energia que um ovo contém, 
permanecem constantes durante o desenvolvimento embrionário segundo outros autores (Pak et al., 1986). De uma maneira geral, a maioria dos trabalhos contradiz a hipótese destes autores, pois para o eulofifeo Edovum puttleri Grissel, houve redução na oviposição e maior mortalidade em ovos mais velhos (Ruberson et al., 1987). De forma análoga, ovos de Helicoverpa zea (Boddie) colocados há mais tempo, propiciaram um maior tempo de desenvolvimento do período ovo-adulto e um menor tamanho de espécimens de T. pretiosum em Anagasta kuehniella (Zeller) (Sá \& Parra, 1994). Assim, para futuros estudos de laboratório com Trichogramma spp. recomenda-se a utilização de ovos de $S$. catenifer com até 60 horas para serem submetidos ao parasitismo.

\section{CONCLUSÕES}

A criação massal de $T$. pretiosum visando ao controle de $S$. catenifer pode ser facilitada com os resultados obtidos na presente pesquisa, quais sejam:

- A relação ideal para se obter o maior parasitismo, em laboratório, é de 1 fêmea de $T$. pretiosum para 20-30 ovos de $S$. catenifer, por um período de exposição de 24 horas;

- Os ovos de $S$. catenifer com até 60 horas de idade são os mais indicados para serem submetidos ao parasitismo por T. pretiosum.

\section{REFERÊNCIAS BIBLIOGRÁFICAS}

FNP CONSULTÓRIA \& COMERCIO. AGRIANUAL 2001: Anuário da Agricultura Brasileira. São Paulo, 2004. 496 p.

GARCIA, M.S. Bioecologia e potencial de controle biológico de Ecdytolopha aurantiana (Lima, 1927) (Lepidoptera: Tortricidae), o bicho-furão-dos-citros, através de Trichogramma pretiosum Riley, 
1879. Piracicaba, 1998. 118 p. Tese (Doutorado) - Escola Superior de Agricultura "Luiz de Queiroz", Universidade de São Paulo.

GOMES, S.M. Comparação de três hospedeiros alternativos para criação e produção massal de Trichogramma pretiosum Riley, 1879 e T. galloi, 1988. Piracicaba, 1997. 106 p. Dissertação (Mestrado) - Escola Superior de Agricultura "Luiz de Queiroz", Universidade de São Paulo. HOHMANN, C.L.; MENEGUIM, A.M. Observações preliminares sobre a ocorrência da broca do abacate, Stenoma catenifer Wals. no estado do Paraná. Anais da Sociedade Entomológica do Brasil, v.22, n. 2, p.417-419, 1993.

HONDA, J.Y.; LUCK, R.F. Interactions between host attributes and wasp size: a laboratory evaluation of Trichogramma platneri as an augmentative biological control agent for two avocado pests. Entomologia Experimentalis et Applicata, v. 100, p.1-13, 2001.

LOPES, J.R.S.; PARRA, J.R.P. Efeito da idade de ovos do hospedeiro natural e alternativo no desenvolvimento e parasitismo de duas espécies de Trichogramma. Revista de Agricultura, v.66, n.3, p.221-241, 1991. NAVA, D.E.; PARRA, J.R.P.; DIEZ-RODRIGUEZ, G.I.; BENTO, J.M.S. Oviposition behavior of Stenoma catenifer Walsingham (Lepidoptera: Elachistidae): chemical and physical stimuli and egg-laying time. Annals of the Entomological Society of America, v.98, p.409-414, 2005.

NAVA, D.E.; PARRA, J.R.P. Biologia de Stenoma catenifer Walsingham (Lepidoptera: Elachistidae) em dietas natural e artificial e estabelecimento de um sistema de criação. Neotropical Entomology, v.34, p.751-759, 2005.

OATMAN, E.R.; MCMURTRY, J.A.; WAGGONNER, M.; PLATNER, G.R.; JOHNSON, H.G. Parasitization of Amorbia cuneana (Lepidoptera: Tortricidae) and Sabulodes aegrotata (Lepidoptera: 
Geometridae) on avocado in southern California. Annals of the Entomological Society of America, v.76, n.1, p.52-53, 1983.

PAK, G.A.; BUIS, C.E.M.; HECK, I.C.C.; HERMANS, M.L.G. Behavioural variations among strains of Trichogramma spp. host age selection. Entomologia Experimentalis et Applicata, v.40, p.247-258, 1986.

PARRA, J.R.P. Técnicas de criação de Anagasta kuehniella, hospedeiro alternativo para produção de Trichogramma. In: PARRA, J.R.P.; ZUCCHI, R.A. (Ed.). Trichogramma e o controle biológico aplicado. Piracicaba: FEALQ, 1997. cap. 4, p.121-150.

PRATISSOLI, D.; PARRA, J.R.P. Seleção de linhagens de Trichogramma pretiosum Riley (Hymenoptera: Trichogrammatidae) para o controle das traças Tuta absoluta (Meyrick) e Phthorimaea operculella (Zeller) (Lepidoptera: Gelechiidae). Neotropical Entomology, v.30, n.2, p.277$282,2001$.

REZNIK, S.Y.; UMAROVA, T.Y.; VOINOVICH, N.D. The influence of previous host age on current host acceptance in Trichogramma. Entomologia Experimentalis et Applicata, v.82, p.153-157, 1997.

RUBERSON, J.R.; TAUBER, M.J.; TAUBER, C.A. Biotypes of Edovum puttleri (Hymenoptera: Eulophidae): responses to developing eggs of the Colorado potato beetle (Coleoptera: Chrysomelidae). Annals of Entomological Society of America, v.80, p.451-455, 1987.

SÁ, L.A.N.; PARRA, J.R.P. Biology and parasitism of Trichogramma pretiosum Riley (Hym., Trichogrammatidae) on Ephestia kuehniella (Zeller) (Lep., Pyralidae) and Heliothis zea (Boddie) (Lep., Noctuidae) eggs. Journal of Applied Entomology, v.118, p.38-43, 1994.

VINSON, S.B. Comportamento de seleção hospedeira de parasitóides de ovos, com ênfase na família Trichogrammatidae. In: PARRA, J.R.P.; 


\section{ZUCCHI, R.A. (Ed.). Trichogramma e o controle biológico aplicado.}

Piracicaba: FEALQ, 1997. cap.3, p.67-120.

WOLFENBARGER, D.O.; COLBURN, B. The Stenoma catenifer, a serious avocado pest. Proceedings of Florida State Horticulture Society, v.92, p.315-318, 1979.

Tabela 1. Número médio de ovos ( \pm DPM) (intervalo de variação) parasitados por fêmea de $T$. pretiosum quando foram oferecidos diferentes números de ovos de $S$. catenifer. Temperatura de $25 \pm 1^{\circ} \mathrm{C}$, UR de $70 \pm 10 \%$ e fotofase de 14 horas

\begin{tabular}{cc}
\hline Número de ovos & Número de ovos parasitados \\
\hline 10 & $9,33 \pm 0,49 \mathrm{~b}(07-10)$ \\
20 & $18,00 \pm 0,52 \mathrm{a}(16-19)$ \\
30 & $23,50 \pm 1,52 \mathrm{a}(17-28)$ \\
40 & $21,83 \pm 1,64 \mathrm{a}(17-28)$ \\
50 & $22,50 \pm 2,62 \mathrm{a}(16-31)$
\end{tabular}

Médias seguidas da mesma letra nas colunas, não diferem estatisticamente entre si, pelo teste de Tukey $(\mathrm{P} \leq 0,05)$.

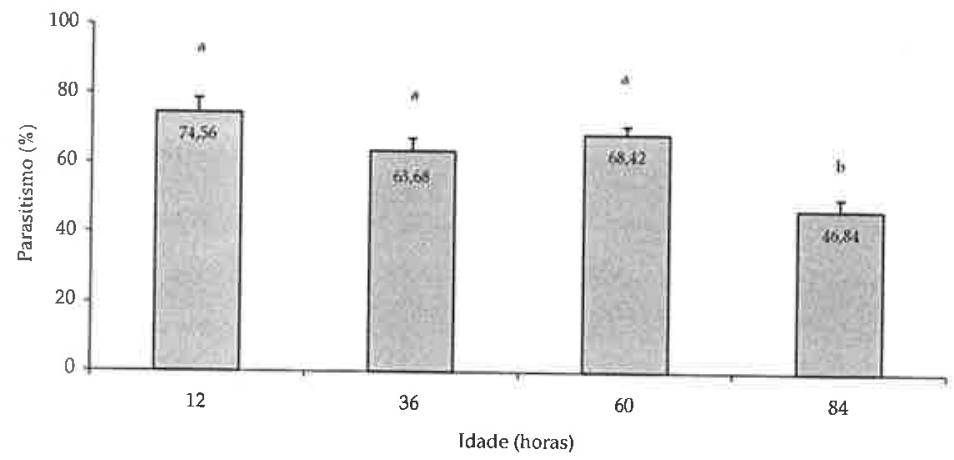

Figura 1. Efeito da idade de ovos de $S$. catenifer sobre a porcentagem de parasitismo de $T$. pretiosum, mantidos em câmaras climatizadas com temperatura de $25 \pm 1{ }^{\circ} \mathrm{C}$, UR de $70 \pm 10 \%$ e fotofase de 14 horas. Colunas seguidas de mesma letra, não diferem estatisticamente entre si, pelo teste de Tukey $(\mathrm{P} \leq 0,05)$ 